\section{Ball lightning an electromagnetic knot?}

SIR - Here we propose a model of ball lightning, a rare and beautiful phenomenon, so far unexplained. Usually, it appears as a flaming ball, most frequently red, orange or bright white, green or blue in some rarer cases, its diameter being typically $10-30 \mathrm{~cm}$, although some examples of a few centimetres or of several metres have also been observed. It moves in an unpredictable way and disappears after several seconds, sometimes smoothly, occasionally with an explosion ${ }^{1,2}$.

Several different theoretical explanations of this phenomenon have been proposed, based on microwaves, combustion, electrical or nuclear processes, or new states of matter - including the opinion that they are no more than optical illusions. No explanation is generally accepted.

Here we propose a solution based on the idea of electromagnetic $\operatorname{knot}^{3,4}$, an electromagnetic field in which any pair of magnetic lines or any pair of electric lines form a link - a pair of linked curves (Fig. 1). Each knot is characterized by two integers $n_{\mathrm{m}}$ and $n_{\mathrm{e}}$, the corresponding magnetic and electric linking numbers (the number of times that each curve crosses a surface bounded by the other). M. Berry (personal communication) suggested that we use them to try to model ball lightning (see also ref. 5).

For simplicity, we will consider here only knots of magnetic type, with no electric field. According to the method explained in refs 3 and 4, we obtain the following magnetic field whose lines are linked $n_{\mathrm{m}}=n$ times

$$
\begin{aligned}
\mathbf{B}= & \frac{4 \sqrt{ } a}{\pi L 2\left(1+R^{2}\right)^{3}}[2(Y-n X Z), \\
& \left.-2(X+n Y Z), n\left(X^{2}+Y^{2}-Z^{2}-1\right)\right]
\end{aligned}
$$

where $(X, Y, Z)=(x, y, z) / L, R^{2}=\left(X^{2}+Y^{2}+Z^{2}\right)=$ $r^{2} / L^{2}, L$ being a length which sets the space scale and $a$ a normalizing constant.

The linking number $n$ is related to the

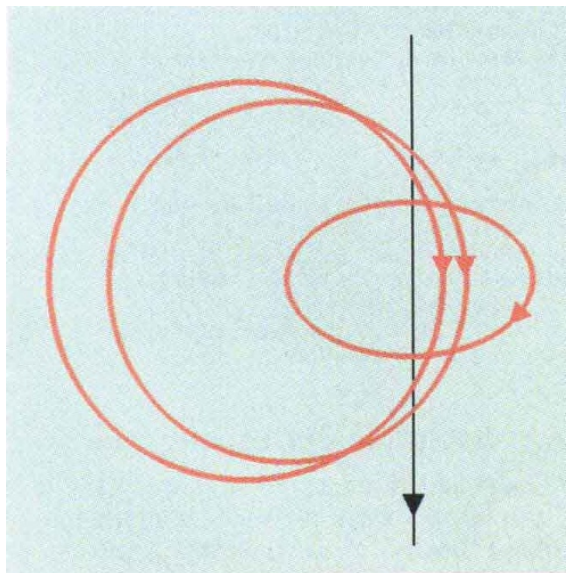

FIG. 1 Schematic representation of the magnetic lines in the case $n=1$. so called magnetic helicity ${ }^{6,7} h$ as

$$
h=\int_{R^{3}} \mathbf{A} \cdot \mathbf{B} \mathrm{d}^{3} x=n a
$$

where $\mathbf{A}$ is the vector potential. This helicity does not depend on $L$ and is a constant of the motion if there is no resistivity (if the conductivity is infinite).

If a magnetic field is coupled to an incompressible plasma that is hot enough, the magnetohydrodynamical approximation, in which the conductivity $\sigma$ is assumed to be infinite, can be used. The motion is described by the Navier-Stokes equation coupled to the Maxwell equation for the magnetic field. A solution of this system is given by $\mathbf{v}= \pm \mathbf{B} / \sqrt{ } \rho, p+B^{2} / 2=$ constant, $\mathbf{v}, \rho$ and $p$ being the velocity, the density and the pressure of the plasma. These states, the streamlines of which are also linked, are not really stationary, as its energy

$$
E=\int\left(\rho \nu^{2} / 2+B^{2} / 2\right) \mathrm{d}^{3} x=\left(n^{2}+1\right) a / L
$$

decreases by increasing the length $L$ (the mean square radius of the energy distribution). Because infinite conductivity implies very high temperature, the knot radiates strongly according to the Stefan-Boltzmann law, which is why it shines and has a flaming aspect. If the knot radiates the same amount as a sphere of radius $L$, its energy loss is $\mathrm{d} E / \mathrm{d} t=$ $-\sigma^{*} 4 \pi^{2} L^{2} T^{4}$, where $\sigma^{*}$ is the Stefan constant and $T$ the temperature, this forces the ball to decrease its energy by increas-

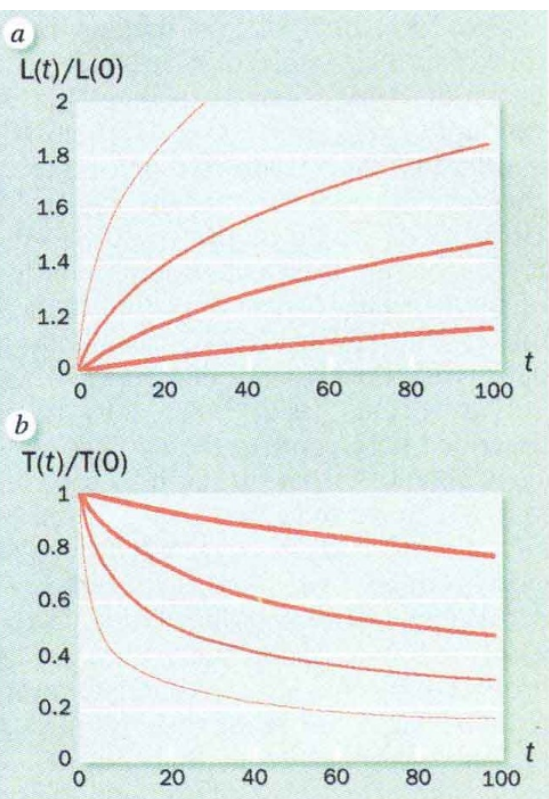

FIG. $2 a, L(t) / L_{0}$ and $b$, temperature $T(t) / T_{0}$ versus time in units of $\tau_{0}$ for infinite conductivity and for the linking numbers $n=0,2,4$ and 10 (in increasing order of thickness). ing $L$ according to equation (3). Assuming that the expansion is adiabatic and that the plasma behaves as an ideal monoatomic gas, its temperature must decrease as $T=\beta L^{-2}$, where $\beta$ is a certain constant coefficient. From equation (3) and the last two relations, it follows that $L=L_{0}\left(1+t / \tau_{\mathrm{n}}\right)^{1 / 5}$, where the characteristic time is $\tau_{n}=\left(n^{2}+1\right) \tau_{0}=\left(n^{2}+1\right)$ $L_{0}^{5} a /\left(20 \pi^{2} \sigma^{*} \beta^{4}\right)$. Consequently, the radius of the ball increases, the magnetic field goes to zero, the temperature decreases as $T=T_{0}\left(1+t / \tau_{n}\right)^{-2 / 5}$, the plasma cools down and the brightness is reduced. The stability of the structure, as measured by the slowness of the cooling, is due to the constraint imposed by the conservation of the helicity equation (2), or equivalently by the linking of the lines. The linking of the knot keeps the plasma hot for a time that increases with $n$ and is longer than for an unlinked system.

We have assumed that the initial temperature is high enough, say above 30,000 $\mathrm{K}$, to imply infinite conductivity. If $\sigma$ could remain infinite, the expansion would continue for ever as $\left(1+t / \tau_{n}\right)^{1 / 5}$. But the conductivity decreases together with the temperature, bringing two new effects into play: first, an additional energy loss by Joule effect which accelerates the cooling; and second, loss of helicity conservation. This leads to the destruction of the knot.

Thus, a magnetic knot formed by a lightning in a storm, where the air is ionized and the temperature very high $(30,000 \mathrm{~K}$ at least), first radiates energy according to the Stefan-Boltzmann law. Insofar as the conductivity can be considered as infinite, the conservation of helicity constrains the decay of the ball, which loses energy by slow expansion as $\left(1+t / \tau_{n}\right)^{1 / 5}$. This produces a reduction of its temperature and a corresponding increase of the resistivity, which in turn produces helicity loss and dissipation of the structure.

To design an experiment to test this model in ball lightning is not easy for obvious reasons. There are two possible laboratory settings: tokamaks ${ }^{8}$ and devices constructed to produce fireballs ${ }^{9}$. Thus it may be possible to test the ideas proposed here in an appropriate experiment.

Antonio F. Rañada, José L. Trueba Departamento de Fisica Teórica, Universidad Complutense, 28040 Madrid, Spain

\footnotetext{
1. Singer, S. Nature 350, 108-109 (1991). 2. Hubert, P., quoted by Gary, C. in La Recherche 211 722-725 (June 1989).

3. Rañada, A. F. J. Phys. A: Math. Gen. 23, L815-L820 (1990).

4. Rañada, A. F. \& Trueba, J. L. Phys. Lett. A 202 337-342 (1995).

5. Kamchatnov, A. M. Sov. Phys. JETP 55, 69-73 (1982)

6. Moffatt, H. K. J. Fluid Mech. 35, 117-129 (1969).

7. Rañada, A. F. Eur. J. Phys. 13, 70-76 (1992).

8. Stenzel, R. L., Urrutia, J. M. \& Rousculp, C. L. Phys.Rev. Lett. 74, 702-705 (1995).

9. Ohtsuki, Y. H. \& Ofuruton, H. Nature 350, 139-141 (1991).
} 